\title{
AtualizaçÃo nO tratamento do tabagismo
}

Autoria: Comissão de Combate ao Tabagismo, Wanderley Marques Bernardo

1. Com relação à eficácia da associação de medicamentos na cessação do tabagismo pode-se afirmar:
a) As combinações não são efetivas quando comparadas a placebo;
b) A combinação pode melhorar o controle dos sintomas de abstinência;
c) As evidências são suficientes para recomendação do uso da terapia combinada;
d) As combinações efetivas são o adesivo de nicotina + vareniclina.

2. Quais as contraindicações e os efeitos colaterais da terapia de reposição de nicotina, bupropiona e vareniclina?
a) Adesivos de nicotina e risco de convulsão;
b) Vareniclina e insuficiência hepática;
c) Goma de nicotina e psoríase;
d) Bupropiona e doença cerebrovascular.

3. Como, quando e em que doses devem ser prescritos os medicamentos que apresentam eficácia na cessação do tabagismo?
a) Adesivos de nicotina de $21 \mathrm{mg}$, passando por $14 \mathrm{mg}$ e depois $7 \mathrm{mg}$ por 6 semanas;
b) Bupropiona de $300 \mathrm{mg}$ duas vezes ao dia por 16 semanas;
c) Nortriptilina de $2 \mathrm{mg}$ a $4 \mathrm{mg}$ ao dia por 12 semanas;
d) Vareniclina de $2 \mathrm{mg}$ duas vezes ao dia por 14 semanas.

4. 0 tratamento farmacológico não está bem estabelecido em fumantes com quais características?

a) Em fumantes que consomem mais de 10 cigarros por dia;

b) Em pacientes com idade superior a 21 anos;

c) Em usuários de outras formas de tabaco;

d) Em pacientes com antecedente de doença cardiovascular.

5. Com relação à extensão do tratamento de tabagismo além de 12 semanas pode-se afirmar que:

a) Fumantes com sintomas de abstinência persistentes podem se beneficiar;

b) 0 uso prolongado ( $\geq 6$ meses) de goma de nicotina não é mais eficaz;

c) 0 uso de bupropiona por período de 2 anos traz benefício;

d) Pacientes com recaída não têm benefício.

Respostas ao Cenário Clínico: Tabagismo (Parte 1) [Publicado na RAMB 2010; 56(2)]

1. A chance de parar de fumar é diferente entre os fumantes que tentam parar sozinhos e aqueles que recebem auxílio de profissionais de saúde e deve-se priorizar o aconselhamento profissional, procedimento este que aumenta a chance de cessação do tabagismo (Alternativa $\mathbf{D}$ ).

2. Avaliações da motivação, do grau de dependência da nicotina, das tentativas prévias de cessação, das recaídas são importantes na abordagem do tabagista durante o tratamento para cessação e deve-se utilizar o questionário de tolerância de Fagerström (Alternativa B).

3. Há influência das técnicas e modelos de aconselhamento e do tempo de realização das mesmas nas taxas de cessação do tabagismo, sendo que, apesar da abordagem intensiva individual e em grupo serem eficazes, alguns estudos apontam para um discreto aumento na taxa de cessação da abordagem individual sobre a de grupo (16,8\% contra $13,9 \%$ ) (Alternativa C).

4. Há diferenças na efetividade do aconselhamento isolado comparado com o uso isolado de medicação e a combinação dos dois tratamentos, sendo a combinação dos dois tratamentos é melhor do que isolados (Alternativa $\mathbf{A}$ ).

5. É controverso se os médicos têm melhores resultados que não-médicos (Alternativa $\mathbf{D}$ ). 